\title{
Automatic Parking Control of Unmanned Vehicle Based on Switching Control Algorithm and Backstepping
}

\author{
Hongbo $\mathrm{Gao}^{1}$, Juping $\mathrm{Zhu}^{2}$, Xinde $\mathrm{Li}^{3}$, Yu Kang ${ }^{2}$, Jiehao $\mathrm{Li}^{4}$ and Hang $\mathrm{Su}^{4}$
}

\begin{abstract}
This paper presents a simple control method for the fully automatic parking of an unmanned vehicle. This method is based on the switching control algorithm and backstepping theory. The fully automatic parking is hard to accomplish since it cannot converge to a specified norm, guarantee the convergence rate, and large uncertainties. Global exponential convergence to any prescribed norm bound ( $\epsilon$-convergence) is guaranteed, and the convergence rate is explicitly given. For the design of steering laws, a method based on backstepping is proposed and analyzed in detail. The backstepping-based design of a multichain system is obtained. Moreover, an exponentially $\epsilon$-convergent control algorithm is adopted to guarantee both converge norm and convergence rate. Real road experiment results are presented and the results show the effectiveness of the control strategies.
\end{abstract}

Index Terms-Automatic parking control, unmanned vehicle, switching control algorithm, backstepping, exponential convergence.

\section{INTRODUCTION}

Most recently, the limited number of vacant parking spots has become a challenging problem for many cities [1-2]. In this situation, how to park a vehicle connected with the robot behavior was widely considered in the nonholonomic motionplanning problem [3]. It is not easy for the drivers to search a bigger spot, and hence they need to be experienced and

This work was supported in part by the Key Research and Development Plan of Anhui Province under Grant No. 202004a05020058, the Fundamental Research Funds for the Central Universities, the Science and Technology Innovation Planning Project of Ministry of Education of China, NVIDIA NVAIL program, the National Natural Science Foundation of China under Grant No. U1804161, and Key Laboratory of Advanced Perception and Intelligent Control of High-end Equipment of Ministry of Education (Anhui Polytechnic University, Wuhu, China, 241000) under Grant No. GDSC202001. And experiments are conducted on NVIDIA DGX-2. (Corresponding author: Hang Su.)

${ }^{1}$ Hongbo Gao is with the Department of Automation, University of Science and Technology of China, Hefei 230026, China, and also with Institute of Advanced Technology, University of Science and Technology of China, Hefei 230088, China, (e-mail: ghb48@ustc.edu.cn).

${ }^{2}$ Juping Zhu and Yu Kang are with the Department of Automation, University of Science and Technology of China, Hefei 230026, China, (email: luguo_qt@163.com; kangduyu@ustc.edu.cn).

${ }^{3} \mathrm{Xinde} \mathrm{Li}$ is with the Key Laboratory of Measurement and Control of CSE, School of Automation, Southeast University, Nanjing 210096, China, and also with the School of Cyber Science and Engineering, Southeast University, Nanjing 210096, China (e-mail: xindeli@seu.edu.cn).

${ }^{4}$ Jiehao $\mathrm{Li}$ and Hang Su are with the Dipartimento di Elettronica, Informazione e Bioingegneria, Politecnico di Milano, Milano 20133, Italy, (e-mail: jiehao.li@mail.polimi.it; hang.su@polimi.it). attentive to park their vehicles. However, even for experienced drivers, their vehicles also suffer from minor scratches. Competition of drivers for a parking spot and the maneuvers of repositioning will increase the possibility of traffic jams. Therefore, this kind of parking is harmful to vehicles and troublesome for drivers.

Many works have been implemented and performed for automatic parking control. The stable analysis of the parking spot was presented in [4] using the Lyapunov function. The steering angle and command duration for executing the parking maneuvers are obtained using the optimization of two parameters [5]. However, the mentioned approaches rely on the gain and parameters so that it is challenging to evaluate the parking performance. An improved sliding mode control scheme was discussed in [6] for the autonomous parking. In [7], a navigation function was introduced. In order to improve the tracking capacity, an improved model associated with the velocity vector was discussed in [8-9]. Furthermore, work in [10] used a method based on a potential field, and it is based on a direction field to ensure the parking. However, the solution's generation requires a lot of calculation time, and the convergence cannot be guaranteed under complex conditions and depends on the optimization parameters.

In the scheme mentioned above, most of the vehicles are operated under the conditions of continuous, longitudinal speed, and steering angle. Therefore, how to deal with the parking problem is the main challenge in real engineering applications. For example, a two-stage path planning method is proposed, and collision-free paths are achieved without considering motion constraints [11-13]. In order to find a feasible path, path planning usually has the characteristic of continuous curvature. A cyclotron curve is designed to solve the collision avoidance and nonholonomic constraint problems [14-15]. However, when the path planning methods are not applicable to automatic parking, otherwise, it will become very complicated.

However, the above control methods do not consider that the model's input is generally required to be continuous and piecewise differentiable, which greatly limits the feasibility of the control. Moreover, many residential quarters, enterprises, and institutions have now adopted a $45^{\circ}$ oblique parking space, which was few considered in previous articles. This paper compares different parking schemes to choose the best parking method, parallel parking, vertical parking, and $45^{\circ}$ oblique parking is considered respectively. The main contributions in 
this article are concluded as below:

1) An exponential $\epsilon$-convergence control algorithm is applied, the convergence bound, and rate problems are solved.

2) According to that, a switching control algorithm is established. The backstepping-based design of a multichain system is obtained. Therefore, the stabilizing feedback gains can be solved directly without solving any equation.

3) A switching control algorithm and backstepping theory are adopted to guarantee that the driving input is free, and the design parameters determine the convergence rate.

This paper is organized as follows: In Section II, the Preliminaries and problem formulation are presented. Section III describes the switching algorithm and introduces the algorithm's application to chained systems and steering laws. In Section IV, the park system is proposed. Section V demonstrates the effectiveness of the automatic parking system via experiment results. Section VI gives the conclusion and future work.

\section{PRELIMINARIES}

\section{A. Vehicle Kinematic Model}

Firstly, a kinematic model of the vehicle is presented [1617]. The Ackermann theory is used to present the front wheels, where $\phi$ represents the steering angle. Define the longitudinal speed as $u(t)$. Particularly, a pure rolling situation is also considered in this article. Thus, the constraints are:

$$
\begin{aligned}
& \dot{x}(t) \sin \Theta(t)-\dot{y}(t) \cos \Theta(t)=0 \\
& \dot{\Theta}(t)=u(t) \tan \phi(t) / l .
\end{aligned}
$$

Respecting the constraints (1) and with the transformation to a path-curvature description, $k_{1}(t)=\tan \phi(t) / l$ and after introducing the required comfort state $k_{2}$ and the acceleration state $k_{3}$, the complete kinematic model $\Sigma$ becomes

$$
\begin{aligned}
& \dot{x}(t)=u(t) \cos \Theta(t), \quad \dot{y}(t)=u(t) \sin \Theta(t) \\
& \dot{\Theta}(t)=u(t) k_{1}(t) \\
& \dot{k}_{1}(t)=k_{2}(t), \dot{k}_{2}(t)=k_{3}(t)
\end{aligned}
$$

subjected to

$$
\left|k_{1}(t)\right| \leq c_{1},\left|k_{2}(t)\right| \leq c_{2},\left|k_{3}(t)\right| \leq c_{3}
$$

where $k_{1}(t)=\tan \phi(t) / l$, and the state $k_{2}$ and $k_{3}$ denote the speed and acceleration, respectively. Noting that $c_{2}$ is based on the actuator. The kinematic model is determined by the trajectory curvature $k_{1}$, derivative $k_{2}$ and acceleration $k_{3}$.

\section{B. Vehicle Dynamics Model}

In this subsection, a dynamic model of the vehicle is considered [18-19], and the model is adopted from the related works in [20]. First, define the center vehicle body as the gravity point, where $(\mathrm{x}, \mathrm{y})$ presents the body coordinate. $u$ denotes the vehicle speed. $\beta$ represents slip angle. Thus, the dynamic model can be described as follows:

$$
\begin{aligned}
{\left[\begin{array}{l}
\dot{\beta} \\
\dot{r}
\end{array}\right] } & =\left[\begin{array}{ll}
a_{11} & a_{12} \\
a_{21} & a_{22}
\end{array}\right]\left[\begin{array}{l}
\beta \\
r
\end{array}\right]+\left[\begin{array}{ll}
b_{11} & b_{12} \\
b_{21} & b_{22}
\end{array}\right]\left[\begin{array}{l}
\phi_{f} \\
\phi_{r}
\end{array}\right] \\
\psi^{*} & =\psi+r \times \Delta t
\end{aligned}
$$

subjected to

$$
\begin{aligned}
& a_{11}=-\frac{C_{f}+C_{r}}{m_{g} u}, a_{12}=-1-\frac{C_{f} l_{f}-C_{r} l_{r}}{m_{g} u^{2}} \\
& a_{21}=-\frac{C_{f} l_{f}-C_{r} l_{r}}{I_{g z}}, a_{22}=-\frac{C_{f} l_{f}^{2}+C_{r} l_{r}^{2}}{I_{g z} u} \\
& b_{11}=\frac{C_{f}}{m_{g} u}, b_{12}=\frac{C_{r}}{m_{g} u} \\
& b_{21}=\frac{C_{f} l_{f}}{I_{g z}}, b_{22}=-\frac{C_{r} l_{r}}{I_{g z}} \\
& C_{f}=\mu c_{f}, C_{r}=\mu c_{r} .
\end{aligned}
$$

When considering the steering in the vehicle parking system, it should be studied the controllability [13]. In other words, the vehicle system is fully controllable in the small-time. It can be used the point set from $q_{\text {start }}$ to a given time $T$. The free configuration state $\mathcal{C}_{\text {free }}$ can be utilized to determine the maneuverability of the system. If the input $\left[k_{1}(t), u(t)\right]$ with $c_{i}>0 \forall i \in\{1,2,3\}$, and $\operatorname{sign}(u(t))$, the dynamic system is small-time controllable [4], [14].

\section{Parking Strategy}

In order to make the vehicle park the designated position well, there is three-step guidance using in the Chinese driving environment [21-22]. The specific steps are summarized as follows:

1) Operate the vehicle directly from the initial position to the desired position in $\left(x_{0}, y_{0}\right)$, and then achieve the angle $\psi$ from the $\mathrm{X}$-axis to the $\mathrm{Y}$-axis.

2) With the movement speed $u$, control the vehicle from the start point to the critical angle position when $\psi(\psi=\Theta)$ from the $\mathrm{X}$-axis to the $\mathrm{Y}$-axis.

3) Operate the vehicle from a critical angle position to the final position, and the angle $\psi$ from the $\mathrm{X}$-axis to the Y-axis converts nearly 0 at the same time.

Therefore, there are five parameters in the three-step parking system, including the initial position $\left(x_{0}, y_{0}\right)$, vehicle speed $u$, steering angle $\phi_{f}$ and yaw angle $\Theta$. Define the length, width and maximum distance parameters, respectively.

\section{Global Exponential $\epsilon$-CONVERGEnCE}

Definition 1: Denote a nonlinear function as $\dot{x}=f(x)$.

1) For $\forall \epsilon>0$, if there exists $T(\epsilon,\|x(0)\|)>0$ and for $\forall$ $t>T$,

$$
\|x(t)\|<\epsilon
$$

is guaranteed, where $\|\cdot\|$ presents the Euclidean norm. Then the nonlinear system is defined as $\epsilon$-convergent.

2) Furthermore, for $\forall t \leq T$, if there exists $k>0$,

$$
\|x(t)\| \leq\|x(0)\| e^{-k t}
$$

is guaranteed for any $x(0)$. Then the nonlinear system is defined as global exponential $\epsilon$-convergence.

\section{A. Feedback Laws}

Noting that the Ackerman kinematic four-wheeled vehicle model is the typical chain system, which can be written as:

$$
\dot{y}_{0}=v, \quad \dot{y}=M y v+N \theta
$$

in which $v$ is the driving input, $\theta$ is the steering input and

$$
y=\left[y_{1}, \ldots, y_{n}\right]^{T}, M=\left[\begin{array}{cc}
0 & I_{n-1} \\
0 & 0
\end{array}\right], N=\left[\begin{array}{l}
0 \\
1
\end{array}\right] \text {. }
$$


Denote the system $\dot{y}=M y v+N \theta$ as y-subsystem. The system is controlled if the driving input $v \neq 0$.

Because of the stability of $(M, N)$, the states $\left(M-N K_{1}\right)$ and $-\left(M-N K_{2}\right)$ are stable, where $K_{1}$ and $K_{2}$ are the feedback states. Define $R_{1}$ and $R_{2}$ as the positive variables. Thus, for $X_{1}, X_{2}>0$, the Lyapunov functions are defined as below:

$$
\left\{\begin{array}{l}
\left(M-N K_{1}\right)^{\mathrm{T}} R_{1}+R_{1}\left(M-N K_{1}\right)+X_{1}=0 \\
-\left(M-N K_{2}\right)^{\mathrm{T}} R_{2}-R_{2}\left(M-N K_{2}\right)+X_{2}=0 .
\end{array}\right.
$$

Theorem 1: Since $\left(M-N K_{1}\right)$ and $-\left(M-N K_{2}\right)$ are stable, we have

1) If $v>0$, let $\theta=-K_{1} y v$, then

$$
\|y(t)\| \leq\|y(0)\| \sqrt{\frac{\lambda_{\max }\left(R_{1}\right)}{\lambda_{\min }\left(R_{1}\right)}} e^{-\frac{\lambda_{\min }\left(X_{1} R_{1}^{-1}\right)}{2}}\left|y_{0}(t)-y_{0}(0)\right| .
$$

2) If $v<0$, let $\theta=-K_{2} y v$, then

$$
\|y(t)\| \leq\|y(0)\| \sqrt{\frac{\lambda_{\max }\left(R_{2}\right)}{\lambda_{\min }\left(R_{2}\right)}} e^{-\frac{\lambda_{\min }\left(X_{2} R_{2}^{-1}\right)}{2}}\left|y_{0}(t)-y_{0}(0)\right| .
$$

Proof: The following is the proof of case $v>0$, and the other case is similar.

The Lyapunov candidate is defined as $V_{1}(y)=y^{\mathrm{T}} R_{1} y$. Combining $\theta=-K_{1} y v$ and equality

$$
\lambda\left(R_{1}^{-1 / 2} X_{1} R_{1}^{-1 / 2}\right)=\lambda\left(X_{1} R_{1}^{-1}\right)
$$

yields

$$
\begin{aligned}
\dot{V}_{1}(y) & =\left(\left(M-N K_{1}\right) y v\right)^{\mathrm{T}} R_{1} y+y^{\mathrm{T}} R_{1}\left(M-N K_{1}\right) y v \\
& =-y^{\mathrm{T}} X_{1} y \cdot|v| \\
& \leq-\lambda_{\min }\left(X_{1} R_{1}^{-1}\right) V_{1}(y) \cdot|v| .
\end{aligned}
$$

Deformation of (14) and integration obtain

$$
V_{1}(y(t)) \leq V_{1}(y(0)) e^{-\lambda_{\min }\left(X_{1} R_{1}^{-1}\right) \int_{0}^{t}|v| d s} .
$$

According to properties

$$
\left\{\begin{array}{l}
\lambda_{\min }\left(R_{1}\right)\|y(t)\|^{2} \leq V_{1}(y) \leq \lambda_{\max }\left(R_{1}\right)\|y(t)\|^{2} \\
\int_{0}^{t}|v(s)| d s=\left|\int_{0}^{t} v(s) d s\right|=\left|y_{0}(t)-y_{0}(0)\right|
\end{array}\right.
$$

the conclusion is guaranteed.

\section{B. Switching Algorithm}

To ensure the boundedness and the stability of $y_{0}$ in chained system, the switching algorithm is proposed as follows:

\section{Convergence Guarantee}

Theorem 2: Based on Theorem 1 and the proposed algorithm, all states are $\epsilon$-convergence.

Proof: Denote the average switching period as $T_{0}$, the total steering time as $q T_{0}$ and the mean of $|v|$ as $\bar{v}$, ie., $\bar{v}=\frac{1}{t} \int_{0}^{t}|v| d s$. Define constants:

$$
\left\{\begin{array}{l}
k=\frac{1}{2} \min \left\{\lambda_{\min }\left(X_{1} R_{1}^{-1}\right), \lambda_{\min }\left(X_{2} R_{2}^{-1}\right)\right\} \\
\tau=\max \left\{\sqrt{\frac{\lambda_{\max }\left(R_{1}\right)}{\lambda_{\min }\left(R_{1}\right)}}, \sqrt{\frac{\lambda_{\max }\left(R_{2}\right)}{\lambda_{\min }\left(R_{2}\right)}}\right\} \\
\gamma=\frac{1}{T_{0}} \ln \tau .
\end{array}\right.
$$

Similar to the proof of Theorem 1,

$$
\|y(t)\| \leq\left\|y\left(t_{i}\right)\right\| \tau e^{-k \int_{t_{i}}^{t}|v(s)| d s}
$$

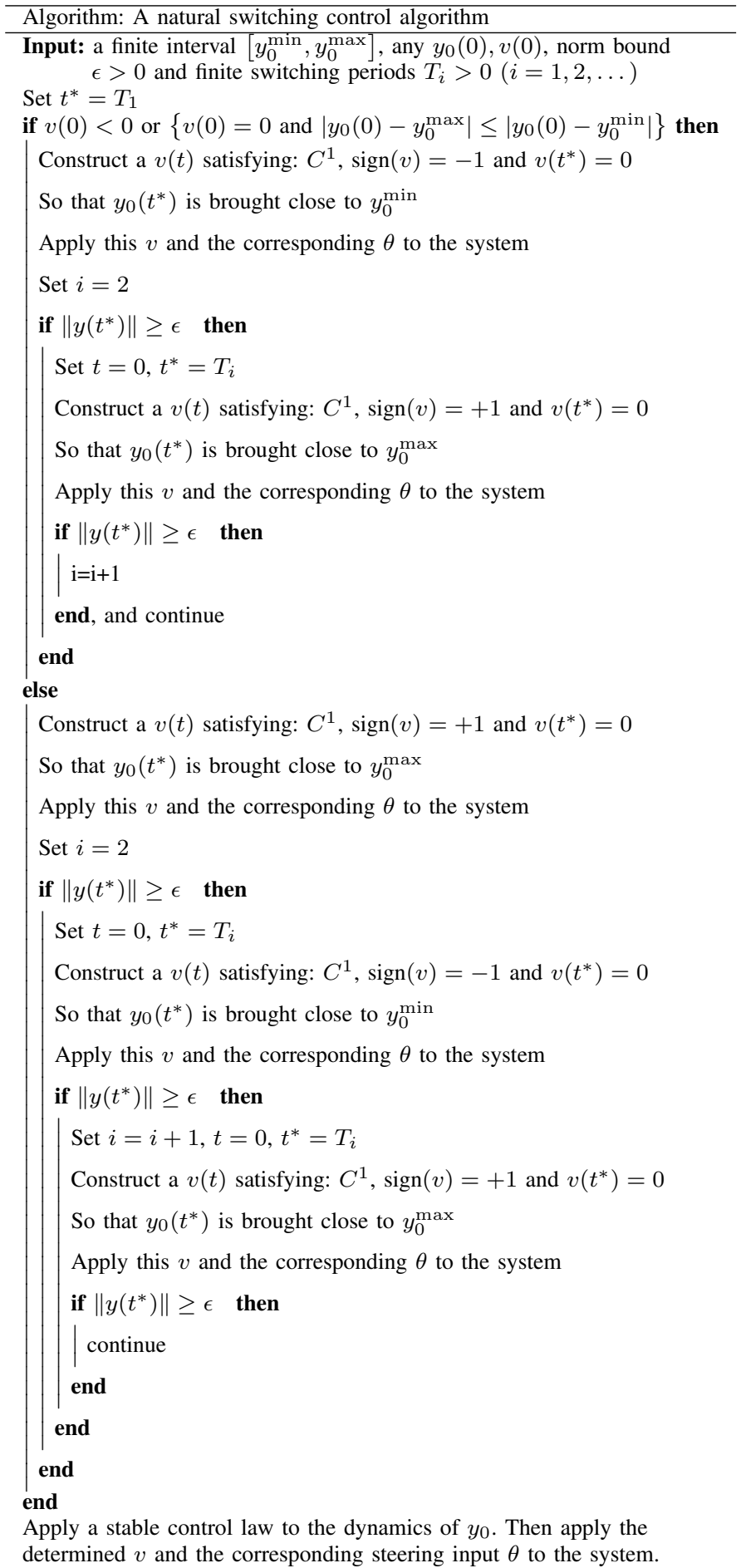

holds in the $i^{\text {th }}$ switching, where $t_{i}=\sum_{j=1}^{i} T_{j}, t_{i}<t \leq t_{i+1}$. Repeating the process, then

$$
\begin{aligned}
\left\|y\left(q T_{0}\right)\right\| & \leq\left\|y\left((q-1) T_{0}\right)\right\| \tau e^{-k \int_{(q-1) T_{0}}^{q T_{0}}|v(s)| d s} \\
& \leq \cdots \leq\|y(0)\| \tau^{q} e^{-k \int_{0}^{q T_{0}}|v(s)| d s} \\
& =\|y(0)\| e^{-(k \bar{v}-\gamma) q T_{0}}
\end{aligned}
$$

ie., for $\forall \epsilon>0$, if $q \geq \ln (\|y(0)\| / \epsilon) /(k \bar{v}-\gamma) T_{0}$, then $\left\|y\left(q T_{0}\right)\right\| \leq \epsilon$. Due to its simple structure, $y_{0}$ is also $\epsilon$ convergence. 
Therefore, the proposed algorithm guarantees global exponential $\epsilon$-convergence of the chained system under the steering laws of Theorem 1 and $\bar{v}>\gamma / k$. If the range of $y_{0}$ is sufficiently enough, the error can be constrained in a reasonable range. Besides, $\bar{v}$ cannot rise too much to avoid the invalidation of the model. The convergence speed is more than $\bar{v} k-\gamma$. Moreover, noticing that the bigger $\bar{v}$ is, the quicker convergence rate will be.

\section{The Backstepping-based Design of One-chain System}

The above subsections guarantee the stability of the system, which is based on Lyapunov equations. In order to get the feedback gains conveniently, the method based on backstepping is presented. And the convergence speed can be clearly given, which depends on the designed parameters.

Definition 2: The linear function $H_{i}\left(y_{1}, \ldots, y_{i}\right)$ is defined as follows:

$$
\begin{aligned}
& x_{1}=y_{1} ; H_{1}\left(y_{1}\right)=\operatorname{sign}(v) k_{1} y_{1} \\
& x_{i}=y_{i}+H_{i-1}\left(y_{1}, \ldots, y_{i-1}\right) \\
& H_{i}\left(y_{1}, \ldots, y_{i}\right)=H_{i-1}\left(y_{2}, \ldots, y_{i}\right)+x_{i-1}+\operatorname{sign}(v) k_{i} x_{i}
\end{aligned}
$$

in which $i=2, \ldots, n$. Thus $x=\left[x_{1}, \ldots, x_{n}\right]^{\mathrm{T}}$ is a linear function of $y$ satisfying $x=A_{ \pm} y . A_{ \pm}$is a lower triangular matrix with diagonal elements of 1 , and $A_{-}$and $A_{+}$are distinguished by the sign of $v$.

Definition 3: For $\forall i=1, \ldots, n$, quadratic function is defined as

$$
I_{i}\left(y_{1}, \ldots, y_{i}\right)=\frac{1}{2}\left(x_{1}^{2}+\cdots+x_{i}^{2}\right)
$$

where the values of $x_{1}, \ldots, x_{i}$ are given in Definition 2 .

Based on Definitions 2 and 3, two feedback gains are constructed.

Lemma:

$$
\|x(t)\| \leq\|x(0)\| e^{-k \int_{0}^{t}|v(s)| d s} .
$$

Proof: Firstly, we prove

$\dot{I}_{i}\left(y_{1}, \ldots, y_{i}\right)=-|v| \sum_{j=1}^{i} k_{j} x_{j}^{2}+x_{i+1} x_{i} v, \quad \forall i \leq n-1$.

1) For $i=1, \dot{x}_{1}=\left[x_{2}-H_{1}\left(y_{1}\right)\right] v$ guarantees

$$
\dot{I}_{1}\left(y_{1}\right)=x_{1}\left[x_{2}-H_{1}\left(y_{1}\right)\right] v=-|v| k_{1} x_{1}^{2}+x_{2} x_{1} v .
$$

2) Assume in the $(i-1)^{\text {th }}$ step:

$$
\dot{I}_{i-1}\left(y_{1}, \ldots, y_{i-1}\right)=-|v| \sum_{j=1}^{i-1} k_{j} x_{j}^{2}+x_{i} x_{i-1} v .
$$

3) For the $i^{\text {th }}$ step: $\dot{I}_{i}=\dot{I}_{i-1}+x_{i} \dot{x}_{i}$ and

$$
\begin{aligned}
\dot{x}_{i} & =\dot{y}_{i}+H_{i-1}\left(\dot{y}_{1}, \ldots, \dot{y}_{i-1}\right) \\
& =y_{i+1} v+H_{i-1}\left(y_{2}, \ldots, y_{i}\right) v
\end{aligned}
$$

$$
\begin{aligned}
& \text { guarantee } \\
& \begin{array}{l}
\dot{I}_{i}\left(y_{1}, \ldots, y_{i}\right) \\
\quad=-|v| \sum_{j=1}^{i-1} k_{j} x_{j}^{2}+x_{i}\left[y_{i+1}+H_{i-1}\left(y_{2}, \ldots, y_{i}\right)+x_{i-1}\right] v \\
=-|v| \sum_{j=1}^{i} k_{j} x_{j}^{2}+x_{i}\left[y_{i+1}+H_{i}\left(y_{1}, \ldots, y_{i}\right)\right] v \\
=-|v| \sum_{j=1}^{i} k_{j} x_{j}^{2}+x_{i+1} x_{i} v .
\end{array}
\end{aligned}
$$

Secondly, for $i=n$,

$$
\begin{aligned}
\dot{x}_{n} & =\dot{y}_{n}+H_{n-1}\left(\dot{y}_{1}, \ldots, \dot{y}_{n-1}\right) \\
& =\theta+H_{n-1}\left(y_{2}, \ldots, y_{n}\right) v
\end{aligned}
$$

guarantees the steering law. Therefore

$\dot{I}_{n}\left(y_{1}, \ldots, y_{n}\right)$

$$
\begin{aligned}
& =-|v| \sum_{j=1}^{n-1} k_{j} x_{j}^{2}+x_{n} \theta+x_{n}\left[H_{n-1}\left(y_{2}, \ldots, y_{n}\right)+x_{n-1}\right] v \\
& =-|v| \sum_{j=1}^{n} k_{j} x_{j}^{2}+x_{n}\left[\theta+H_{n}\left(y_{1}, \ldots, y_{n}\right) v\right] \\
& =-|v| \sum_{j=1}^{n} k_{j} x_{j}^{2} \leq-2 k|v| I_{n}
\end{aligned}
$$

i.e., the Lemma holds.

Theorem 3: Assume $v$ has the same sign and $k_{i}>0, \forall i$. Based on the steering law of $\theta=-H_{n}\left(y_{1}, \ldots, y_{n}\right) v$,

$$
\|y(t)\| \leq\|y(0)\| \varsigma e^{-k \int_{0}^{t}|v(s)| d s}
$$

holds, where $k=\min _{1 \leq i \leq n}\left\{k_{i}\right\}>0$,

$$
\varsigma=\max \left\{\sqrt{\frac{\lambda_{\max }\left(A_{+}^{\mathrm{T}} A_{+}\right)}{\lambda_{\min }\left(A_{+}^{\mathrm{T}} A_{+}\right)}}, \sqrt{\frac{\lambda_{\max }\left(A_{-}^{\mathrm{T}} A_{-}\right)}{\lambda_{\min }\left(A_{-}^{\mathrm{T}} A_{-}\right)}}\right\} .
$$

Proof: Property

$$
\lambda_{\min }\left(A_{ \pm}^{\mathrm{T}} A_{ \pm}\right)\|y(t)\|^{2} \leq\|x\|^{2} \leq \lambda_{\max }\left(A_{ \pm}^{\mathrm{T}} A_{ \pm}\right)\|y(t)\|^{2}
$$

guarantees

$$
\begin{aligned}
\lambda_{\min }\left(A_{ \pm}^{\mathrm{T}} A_{ \pm}\right)\|y(t)\|^{2} & \leq\left\|A_{ \pm} y(0)\right\|^{2} e^{-2 k \int_{0}^{t}|v(s)| d s} \\
& \leq\|y(0)\|^{2} \lambda_{\max }\left(A_{ \pm}^{\mathrm{T}} A_{ \pm}\right) e^{-2 k \int_{0}^{t}|v(s)| d s}
\end{aligned}
$$

therefore $\|y(t)\| \leq\|y(0)\| \varsigma e^{-k \int_{0}^{t}|v(s)| d s}$ holds.

\section{E. The Backstepping-based Design of Multichain System}

Similarly, $H_{n_{j} j}\left(y_{1 j}, \ldots, y_{n_{j} j}\right)$ is defined as follows,

$$
\begin{aligned}
& x_{1 j}=y_{1 j} ; H_{1 j}\left(y_{1}\right)=\operatorname{sign}(v) k_{1 j} y_{1 j} \\
& x_{i j}=y_{i j}+H_{(i-1) j}\left(y_{1 j}, \ldots, y_{(i-1) j}\right) \\
& H_{i j}\left(y_{1 j}, \ldots, y_{i j}\right)=H_{(i-1) j}\left(y_{2 j}, \ldots, y_{i j}\right)+x_{(i-1) j}+\operatorname{sign}(v) k_{i j} x_{i j}
\end{aligned}
$$

in which $j=1, \ldots, m$, and $A_{i, \pm}$ presents $A$ in Definition 2 for the $i^{\text {th }}$.

Theorem 4: Assume that $v$ does not change its sign and $k_{i j}>0, \forall i, j$. Based on the steering laws, the multichain system satisfies

$$
\|y(t)\| \leq\|y(0)\| \varsigma e^{-k \int_{0}^{t}|v(s)| d s}
$$


where $\varsigma_{j}=\max \left\{\sqrt{\frac{\lambda_{\max }\left(A_{j,+}^{\mathrm{T}} A_{j,+}\right)}{\lambda_{\min }\left(A_{j,+}^{\mathrm{T}} A_{j,+}\right)}}, \sqrt{\frac{\lambda_{\max }\left(A_{j,-}^{\mathrm{T}} A_{j,-}\right)}{\lambda_{\min }\left(A_{j,-}^{\mathrm{T}} A_{j,-}\right)}}\right\}$, $k_{j}=\min _{1 \leq i \leq n_{j}}\left\{k_{i j}\right\}, \varsigma=\max _{1 \leq j \leq m} \varsigma_{j}, k=\min _{1 \leq j \leq m} k_{j}$.

Proof: Similar to Theorem 3, we have: $\forall j=1, \ldots, m$

$$
\begin{gathered}
\left\|\left[y_{1 j}(t), \ldots, y_{n_{j} j}(t)\right]\right\| \leq\left\|\left[y_{1 j}(0), \ldots, y_{n_{j} j}(0)\right]\right\| \\
\cdot \varsigma_{j} e^{-k_{j} \int_{0}^{t}|v(s)| d s} .
\end{gathered}
$$

Therefore,

$$
\begin{aligned}
\|y(t)\|^{2} & =\sum_{j=1}^{m}\left\|\left[y_{1 j}(t), \ldots, y_{n_{j} j}(t)\right]\right\|^{2} \\
& \leq e^{-2 k \int_{0}^{t}|v(s)| d s} \varsigma^{2} \sum_{j=1}^{m}\left\|\left[y_{1 j}(0), \ldots, y_{n_{j} j}(0)\right]\right\|^{2} \\
& =\|y(0)\|^{2} \varsigma^{2} e^{-2 k \int_{0}^{t}|v(s)| d s} .
\end{aligned}
$$

Combining the proposed algorithm and Theorem 4, the global exponential $\epsilon$ - convergence of chained system (with a rate greater than $\bar{v} k-\gamma$ ) and the stability of state $y_{0}$ is guaranteed if $\bar{v}>\gamma / k$. As shown in Theorems 3 and 4, the proposed method guarantees convergence every time.

\section{Automatic Parking Systems}

The method designed in this paper can perform the parking task in any parking scene, but the efficiency is not high because the vehicle needs to move back and forth many times to park in the garage. Therefore, this paper introduces suitable auxiliary parking destinations for three common parking scenes: parallel parking, vertical parking and $45^{\circ}$ oblique parking, so as to improve parking efficiency. Similar paths can also be designed for other parking scenes. For common parking scenes, two or three schemes are proposed and compared, based on the criterion of "lower lane width of the straight stage before warehousing and size of the parking space require better method". Related parameters are shown in Table I.

TABLE I

The Parameter of Zhihong UnManNed Vehicle and Parking SPACE

\begin{tabular}{ccc}
\hline \hline Symbols & Meaning & Value \\
\hline$L_{1}$ & Full length of vehicle & $3200[\mathrm{~mm}]$ \\
$L_{2}$ & Width of vehicle & $1670[\mathrm{~mm}]$ \\
$L_{3}$ & Wheelbase & $2150[\mathrm{~mm}]$ \\
$d$ & Safety margin determined by designer & $150[\mathrm{~mm}]$ \\
$d_{1}$ & Distance from rear wheel axle center to rear of vehicle & $485[\mathrm{~mm}]$ \\
$\Phi_{\max }$ & Maximum steering angle of front wheel & $25^{\circ}$ \\
$R_{\min }$ & Minimum radius of rotation at rear wheel center & $4550[\mathrm{~mm}]$ \\
\hline & & $150[\mathrm{~mm}]$ \\
$d_{2}$ & Width of the line of three parking space & $220[\mathrm{~mm}]$ \\
& & $150[\mathrm{~mm}]$ \\
\cline { 3 - 3 } & & $6150[\mathrm{~mm}]$ \\
$L_{G, 1}$ & Length of three parking space including line & $4990[\mathrm{~mm}]$ \\
& & $5690[\mathrm{~mm}]$ \\
\cline { 3 - 4 } & & $2460[\mathrm{~mm}]$ \\
$L_{G, 2}$ & Width of three parking space including line & $2500[\mathrm{~mm}]$ \\
& & $2670[\mathrm{~mm}]$ \\
\hline \hline
\end{tabular}

\section{A. Parallel Parking}

For parallel parking, four methods are designed, according to the following two criteria: the front or rear of the vehicle enters the parking space firstly, there are two or three main steps. It can be proved that the method that the rear of the vehicle enters the parking space firstly with two steps is better. This method can be specifically described as follows:

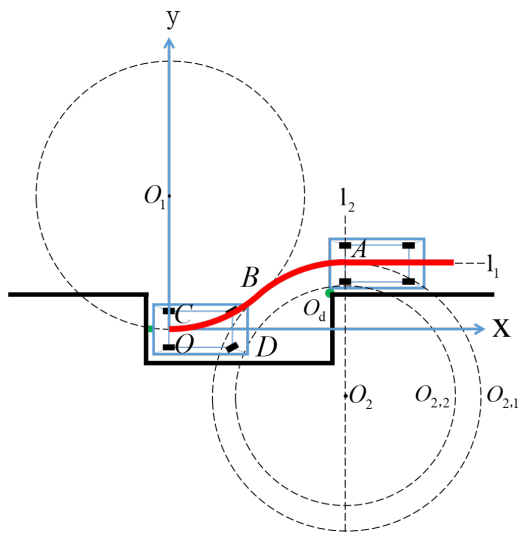

Fig. 1. The first method of parallel parking.

1) As in Fig. 1, the green figures present the safety margin. Using $L_{2}, L_{G 2}, d, d_{1}$ and under the condition that the distances from point $O$ to both sides of parking space are equal, the position of point $O$ can be determined, which presents the final position of the vehicle. The center coordinate can be defined as $O(x, y)$. Point $O$ is also denoted as $C$. Determine a circle $O_{1}$ with radius $R_{\text {min }}$ centered at $\left(0, R_{\min }\right)$, which guarantees the rotation is realizable and simplest, the steering angle is maximum. Denote the inside point of the front of the vehicle in the final parking position as $D$. The red circular arc of circle $O_{1}$ represents one of the reversing steps.

2) Before entering the warehouse, the body is parallel to the garage. In order to minimize the requirement for lane width, two lines $l_{1}$ and $l_{2}$ can be obtained first, which is respectively parallel to the $x$-axis and the $y$-axis at the center of the two rear wheels of the vehicle in the initial position. Second, concentric circles $\mathrm{O}_{2}$ centered at line $l_{1}$ is obtained, satisfying that the bigger circle $O_{2,1}$ is tangential to line $l_{1}$ at point $A$ with radius $R_{\min }$ and the smaller circle $\mathrm{O}_{2,2}$ crosses the rear inside corner.

3) Now determine the specific location of concentric circles $\mathrm{O}_{2}$ and the initial position.

(i) The circle $O_{2,1}$ is tangential to the circle $O_{1}$ at point $B$, guaranteeing two consecutive turns at $B$ can be realized.

(ii) The circle $O_{2,2}$ is tangential to the circle $O_{d}$, which is centered at the outside corner of parking space with radius $d$. This ensures the first turn $A-B$ does not touch the line.

(iii) The inside boundary line of the vehicle is located above the circle $O_{d}$. This ensures the safety of straight travel before warehousing.

The parking path can be simply expressed as $A-B-C$, i.e., the red line in Fig. 1. The vehicle is required to not touch the garage in the process of $B-C$, which means point $D$ will not touch circle $O_{d}$. In fact, the general parallel parking 
space can meet this condition. So that safety is ensured in the whole designed route.

From the above paragraph, the related parameters in $O(x, y)$ are obtained through geometric computations as follows:

$$
\left\{\begin{array}{l}
O_{d}\left(x_{d}, y_{d}\right) \\
O_{2}\left(x_{0}, y_{0}\right) \\
A\left(x_{0}, y_{0}+R_{\min }\right) \\
B\left(x_{0} / 2,\left(y_{0}+R_{\min }\right) / 2\right) \\
C(0,0) \\
D\left(L_{1}-d_{1},-L_{2} / 2\right) \\
\Theta_{B}=\tan ^{-1} x_{0} /\left(R_{\min }-y_{0}\right)
\end{array}\right.
$$

in which $x_{d}=L_{G, 1}-d-d_{1}-2 d_{2}, y_{d}=L_{G, 2} / 2, x_{0}$ and $y_{0}$ are the solutions of following equations, satisfying $y_{0}<0$ :

$$
\left\{\begin{array}{l}
x_{0}^{2}+\left(y_{0}-R_{\min }\right)^{2}=4 R_{\min }^{2} \\
\left(x_{0}-x_{d}\right)^{2}+\left(y_{0}-y_{d}\right)^{2}=\left(R_{O_{2,2}}-d\right)^{2}
\end{array}\right.
$$

in which $R_{O_{2,2}}=\sqrt{d_{1}^{2}+\left(R_{\min }-L_{2} / 2\right)^{2}}$.

The detailed control process is as follows:

1) Establish two rectangular coordinate systems with the origin at $A$ and $B$, and each $x$-axis is tangent to circle $\mathrm{O}_{2,1}$.

2) Use the designed steering input, let the vehicle pass through the point $A$, point $B$, and point $C$ automatically along the designed route.

3) Utilize the switching algorithm until the stopping accuracy is satisfactory.

This method is better than the other three methods based on the narrated criterion. To see this clearly, we give the situation of the front of the vehicle entering the garage with three main steps, as shown in Fig. 2. The other two situations are similar.

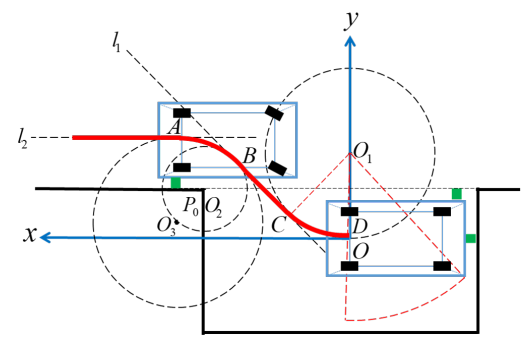

Fig. 2. The second method of parallel parking.

1) As shown in Fig. 2, the position of point $O$ can be determined easily. Establish a similar rectangular coordinate system $O(x, y)$. Point $O$ is also denoted as $D$. Circle $O_{1}$ is centered at $\left(0, R_{\min }\right)$ with radius $R_{\min }$.

2) We expect to finish the parallel parking by three main steps. As in Fig. 2, circle $\mathrm{O}_{2}$ is centered at the outside corner $P_{0}$ with radius $\frac{L_{2}}{2}+d$.

3) Determine the circle $\mathrm{O}_{3}$, which is tangential to the line $l_{1}$ at point $B$ and the radius is $R_{\min }$. The line $l_{2}$ is tangential to the circle $O_{3}$ at point $A$ and parallel to the $x$-axis. The distance from the straight line $l_{2}$ to the garage line is $L_{2} / 2+d$, and the line is the central vehicle axis in the starting position.

4) Therefore, the parking path can be simply expressed as $A-B-C-D$.
In Fig. 2, the red dotted lines show that the parking space's width needs to be large enough to guarantee this turning process without touching line.

\section{B. Vertical Parking}

For vertical parking, two methods are presented. The method shown in Fig. 3 will be introduced specifically:

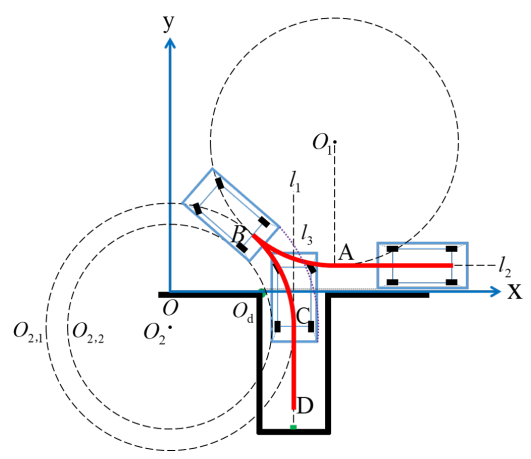

Fig. 3. The first method of vertical parking.

1) Line $l_{1}$ is the axisymmetric line of parking space.

2) Denote the point across line $l_{1}$ and the distance $d_{1}+d$ from the garage as $D$, which presents the vehicle's final position.

3) Similar to Fig. 1, there are also circles $O_{d}, O_{2}, O_{2,1}$, $O_{2,2}$, but circle $O_{2,1}$ is tangent to line $l_{1}$ at point $C$ and circle $O_{2,2}$ is tangent to circle $O_{d}$. Determine the circular arc $l_{3}$, taking the center of circle $\mathrm{O}_{2}$ as the center and the radius is long enough to across the outside corner of the vehicle rear as the purple dotted line. The vehicle is required not to touch the garage in the process of $B-C$, which means circular arc $l_{3}$ will not touch the parking spaceline. In fact, the general vertical parking space can meet this condition.

4) Denote the point as $O$, which is on the outside line of parking space and above the center of circle $\mathrm{O}_{2}$. The center coordinate is $O(x, y)$.

5) Line $l_{2}$ is across the axisymmetric line of the vehicle. The vehicle is parallel to the outside line of the parking space and their distance is the safety margin $d$.

6) Determine the circle $O_{1}$ with radius $R_{\min }$. The circle $O_{1}$ is tangent to the circle $O_{2,1}$ at point $B$ and tangent to the line $l_{2}$ at point $A$.

7) Therefore, the safety is ensured in the whole designed route, and the parking path can be simply expressed as $A-B-C-D$.

From the above paragraph, the related parameters in $O(x, y)$ are obtained through geometric computations:

$$
\left\{\begin{array}{l}
O_{1}\left(x_{2}, y_{2}+R_{\min }\right) \\
O_{2}\left(0,-h_{0}\right) \\
O_{d}\left(R_{\min }+d_{2}-L_{G, 2} / 2,0\right) \\
A\left(x_{2}, y_{2}\right) \\
B\left(x_{2} / 2,\left(y_{2}+R_{\min }-h_{0}\right) / 2\right) \\
C\left(R_{\min },-h_{0}\right) \\
D\left(R_{\min }, d+d_{1}+d_{2}-L_{G, 1}\right) \\
\Theta_{B}=\tan ^{-1}\left(y_{2}+R_{\min }+h_{0}\right) / x_{2}+90^{\circ}
\end{array}\right.
$$


in which

$$
\left\{\begin{array}{l}
R_{O_{2,2}}=\sqrt{d_{1}^{2}+\left(R_{\min }-L_{2} / 2\right)^{2}} \\
h_{0}=\sqrt{\left(R_{O_{2,2}}-d\right)^{2}-\left(R_{\min }+d_{2}-L_{G, 2} / 2\right)^{2}} \\
x_{2}=\sqrt{4 R_{\min }^{2}-\left(y_{2}+R_{\min }+h_{0}\right)^{2}} \\
y_{2}=d+L_{2} / 2
\end{array}\right.
$$

The parking control procedure is similar and the specific process of the second method is as follows:

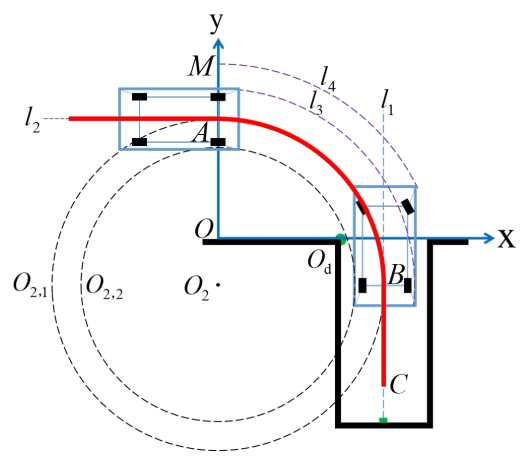

Fig. 4. The second method of vertical parking.

1) Similar to the circles $O_{d}, O_{2,1} O_{2,2}$, line $l_{1}$, point $B$, $C$ and a coordinate system $O(x, y)$ in the Fig. 2, there are also circles, line, points and coordinate system as in Fig. 4.

2) Determine the line as $l_{2}$, which is parallel to the $x$ axis and tangent to circle $\mathrm{O}_{2}$ at point $A$. Therefore, the parking path can be simply expressed as $A-B-C$.

3) Determine the circular arc $l_{3}$, take the center of circle $\mathrm{O}_{2}$ as the center, and the radius is long enough to across the outside corner of the vehicle rear. The vehicle must not touch the garage the process of $A-B$, which means circular arc $l_{3}$ will not touch the parking spaceline.

4) Determine the circle $l_{4}$, take the center of circle $\mathrm{O}_{2}$ as the center and the radius is long enough to across the outside corner of the vehicle head. The point $M$ is the standard of lane width requirements, which is the intersection of $y$-axis and line $l_{4}$. So that the method of Fig. 3 is better than that of Fig. 4.

\section{C. $45^{\circ}$ Oblique Parking}

For $45^{\circ}$ oblique parking, the given parameters are similar, however $L_{G, 1}$ presents the length of the longer diagonal line, and the $L_{G, 2}$ presents the distance between two diagonal lines. Three methods are presented. The method shown in Fig. 5 will be introduced specifically:

1) Line $l_{1}$ is the axisymmetric line of parking space.

2) Similar to Fig. 3, there are also circles $\mathrm{O}_{d}, \mathrm{O}_{2}, \mathrm{O}_{2,1}$, $O_{2,2}$ and line $l_{2}$, but with circle $O_{2,1}$ is tangent to line $l_{1}$ at point $C$ and circle $O_{2,2}$ is tangent to circle $O_{d}$ as in Fig. 5.

3) Denote the point as $O$, which is on the outside corner of the parking space.

4) Determine the circle $O_{1}$ with radius $R_{\min }$, and circle $O_{1}$ is tangent to the circle $O_{2,1}$ at point $B$, tangent to the line $l_{2}$ at point $A$.

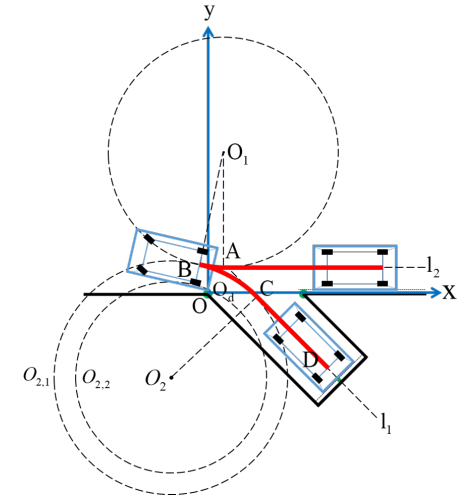

Fig. 5. The first method of $45^{\circ}$ oblique parking.

5) Similar to Fig. 3, the vehicle is required to not touch the garage in the process of $B-C$, and the condition is mostly satisfied for $45^{\circ}$ oblique parking. Therefore, safety is ensured in the whole designed route. The parking path can be simply expressed as $A-B-C-D$.

From the above paragraph, the related parameters in $O(x, y)$ are obtained through geometric computations as

$$
\left\{\begin{array}{l}
O_{1}\left(x_{0}, y_{0}\right) \\
O_{2}\left(x_{1}, y_{1}\right) \\
A\left(x_{0}, y_{0}-R_{\min }\right) \\
B\left(\left(x_{0}+x_{1}\right) / 2,\left(y_{0}+y_{1}\right) / 2\right) \\
C\left(\frac{\sqrt{2}\left(x_{1}-y_{1}\right)+L_{G, 2}-2 d_{2}}{2 \sqrt{2}}, \frac{\sqrt{2}\left(y_{1}-x_{1}\right)+L_{G, 2}-2 d_{2}}{2 \sqrt{2}}\right) \\
D\left(x_{2}, y_{2}\right) \\
\Theta_{B}=\tan ^{-1}\left(y_{0}-y_{1}\right) /\left(x_{0}-x_{1}\right)+90^{\circ}
\end{array}\right.
$$

in which $x_{1}$ and $y_{1}$ are the solutions of following equations and $y_{1}$ takes the smaller solution:

$$
\left\{\begin{array}{l}
x_{1}^{2}+y_{1}^{2}=\left(R_{O_{2,2}}-d\right)^{2} \\
x_{1}+y_{1}-\sqrt{2} L_{G, 2} / 2+\sqrt{2} d_{2}=-\sqrt{2} R_{\min }
\end{array}\right.
$$

where

$$
\left\{\begin{array}{l}
R_{O_{2,2}}=\sqrt{d_{1}^{2}+\left(R_{\min }-L_{2} / 2\right)^{2}} \\
y_{0}=R_{\min }+L_{2} / 2+d \\
x_{0}=x_{1}+\sqrt{4 R_{\min }^{2}-\left(y_{0}-y_{1}\right)^{2}} \\
y_{2}=\left(-L_{G, 1}+L_{G, 2} / 2+d+d_{1}+d_{2}\right) / \sqrt{2} \\
x_{2}=\sqrt{2} L_{G, 2} / 2-y_{2}-\sqrt{2} d_{2} .
\end{array}\right.
$$

The parking control procedure is similar. Two other methods

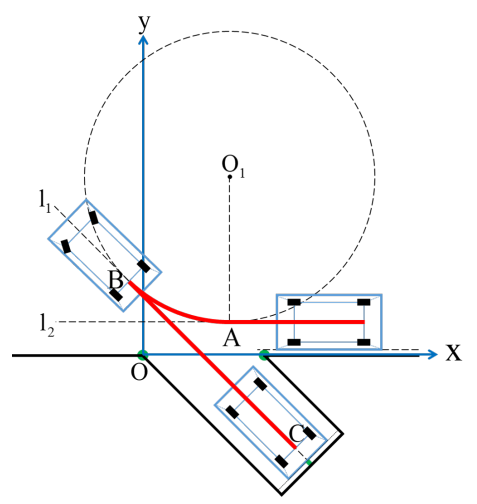

Fig. 6. The second method of $45^{\circ}$ oblique parking. 
for $45^{\circ}$ oblique parking, one is similar to Fig. 5 , and the other is similar to Fig. 4. Because these two methods are not better than Fig. 5 with similar reasons to vertical parking, so we will just briefly describe them.

For the method similar to Fig. 5, as shown in Fig. 6.

1) Line $l_{1}$ is the axisymmetric line of parking space.

2) Similar to Fig. 5, there are also line $l_{2}$, point $C$ and a coordinate system $O(x, y)$.

3) Determine the circle $O_{1}$ with radius $R_{\text {min }}$, and circle $O_{1}$ is tangent to the line $l_{1}$ at point $B$, tangent to the line $l_{2}$ at point $A$. Therefore, the designed path can be simply expressed as $A-B-C$.

For the method similar to Fig. 4, as shown in Fig. 7.

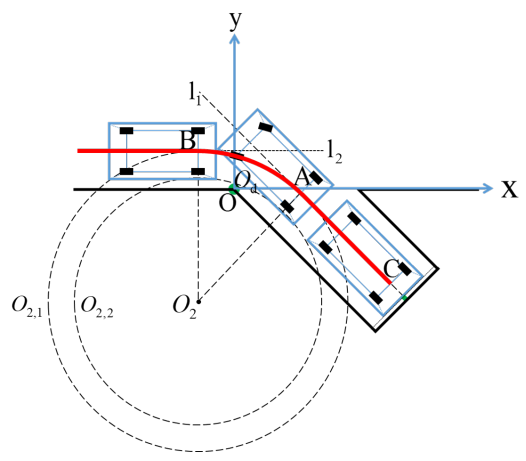

Fig. 7. The third method of $45^{\circ}$ oblique parking.

1) Line $l_{1}$ is the axisymmetric line of parking space.

2) Similar to Fig. 4 , there are also point $C$, line $l_{1}$, circles $O_{d}, O_{2,1} O_{2,2}$ but the circle $O_{2,1}$ is tangent to line $l_{1}$ at point $B$.

3) Denote a similar coordinate system as $O(x, y)$. Point $O$ is also the center of circle $O_{d}$.

4) Determine the line $l_{2}$, which is parallel to $x$-axis and tangent to the circle $O_{2,1}$ at the point $A$. Therefore, the designed path can be simply expressed as $A-B-C$.

\section{EXPERIMENT AND RESUlts ANALYSis}

\section{A. Experiment Setup}

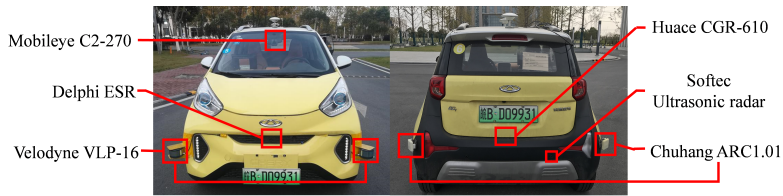

Fig. 8. Zhihong unmanned vehicle.

In the three parking experiments, the used vehicle is Zhihong unmanned vehicle. The sensor deployment of the Zhihong unmanned vehicle is shown in Fig. 8, which consists of 1 vision sensor, seven radar sensors and one integrated position/attitude sensor. The vision sensor is a camera (Mobileye C2-270), equipped on the frontal mirror's back. The radar sensors include $1 \mathrm{MMW}$ (Millimeter-wave) radar (Delphi ESR), 2 sixteen-line laser radars (Velodyne VPL-16), 2 ultrasonic radars $(\operatorname{Softec}(\mathbb{R})$ ) and 2 MMW (Millimeter-wave) radars (Chuhhang ARC1.01). The integrated position/attitude sensor includes a global positioning system (GPS) and an inertial navigation system (INS), which is from Huace $\mathbb{R}$.

In these experiments, different feedback gains are used in the stability control for each auxiliary destination as well as the final destination. For the sake of safety, the Zhihong unmanned vehicle started the collision avoidance mode. The relevant experimental parameters are shown in table I. The safe margin of $d$ in these experiments is designed as $150 \mathrm{~mm}$.

\section{B. Experiment Results and Analysis}

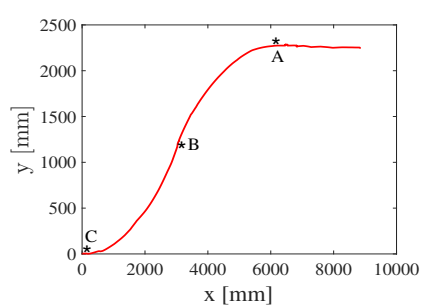

(a)

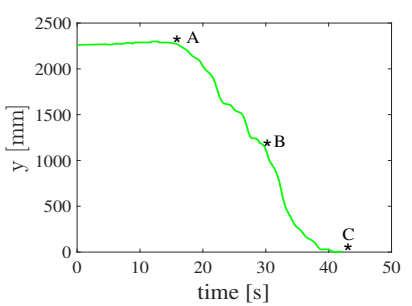

(c)

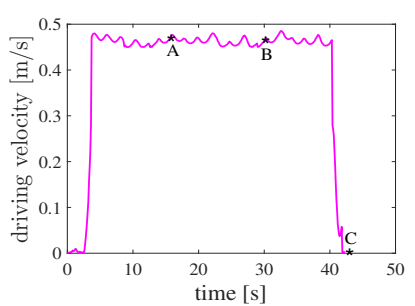

(e)

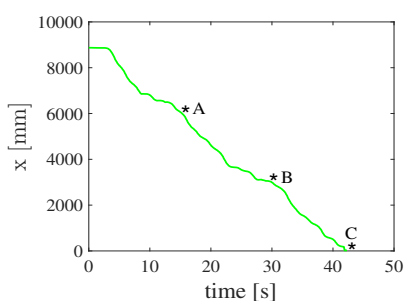

(b)

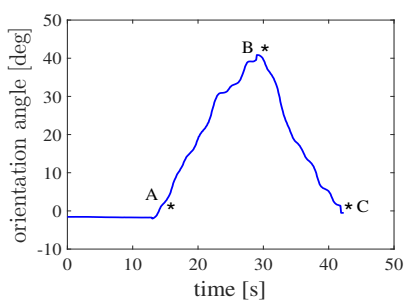

(d)

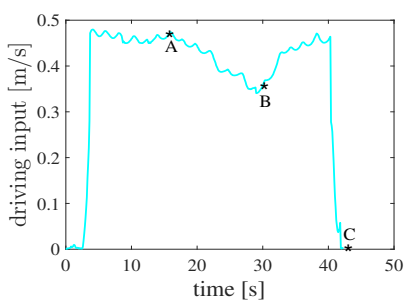

(f)
Fig. 9. The result of parallel parking experiment. (a) $x-y$ path, (b) $x$ coordinate, (c) $y$ coordinate, (d) orientation angle $\Theta$, (e) driving velocity $u$, (f) driving input: $u * \cos \Theta$.

Compared with the simulation experiment, the real experiment mainly has the following system errors:

1) The certain errors between the theoretical and actual parameters of the vehicle;

2) The data measured by the sensor has error;

3) The actual operation cannot reach the ideal level, for example, actual operation has inertia delay.

The experimental results are shown in Figs. 9, 10, and 11, which are marked with key points. They are the theoretical values of the auxiliary destinations and the final destination.

For parallel parking, the experimental result is shown in Fig. 9. Comparing experimental diagram Fig. 9 (a) with the theoretical diagram Fig. 1, it can be concluded that the experiment is ideal. The curve in Fig. 9 (a) is smooth and has a small deviation at points $\mathrm{A}, \mathrm{B}$ and $\mathrm{C}$, so the system 


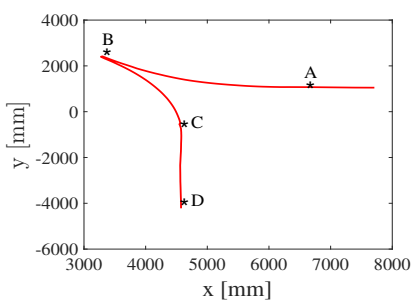

(a)

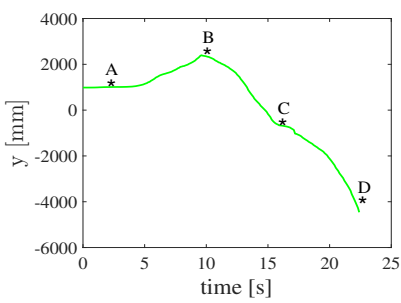

(c)

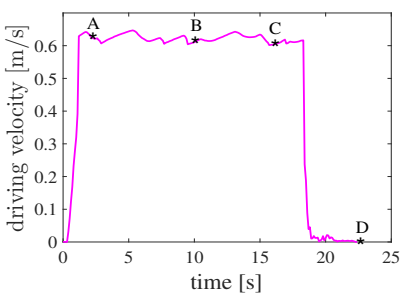

(e)

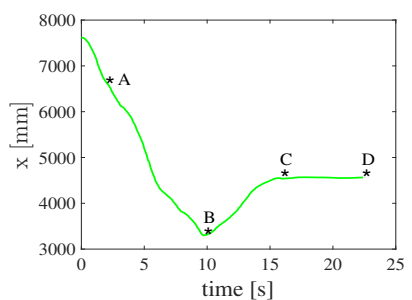

(b)

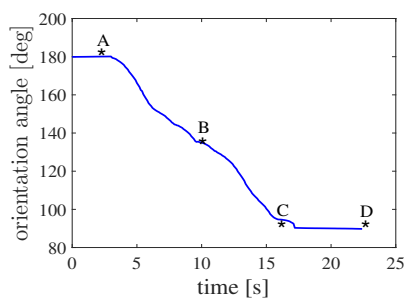

(d)

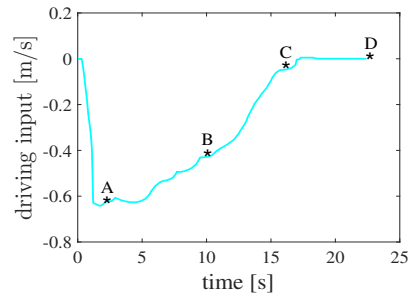

(f)

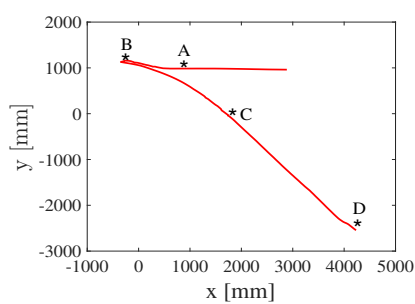

(a)

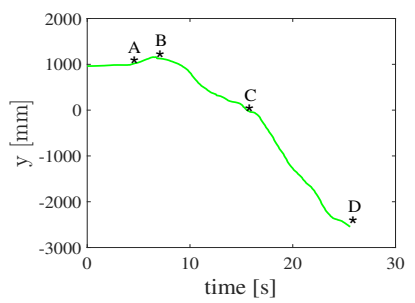

(c)

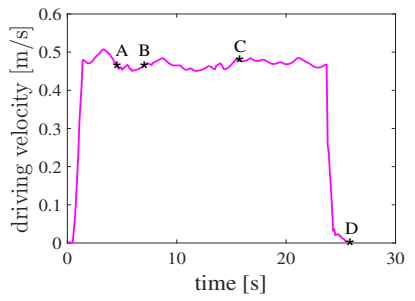

(e)

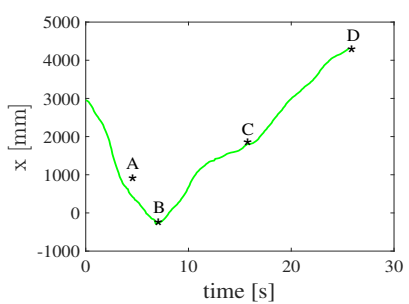

(b)

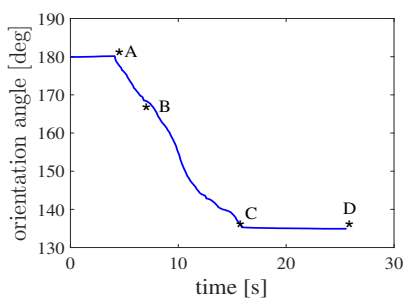

(d)

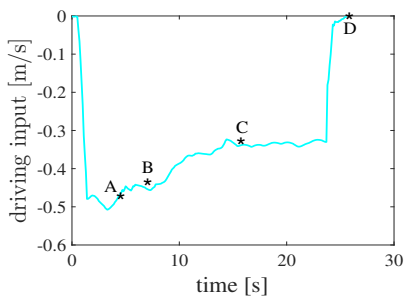

(f)
Fig. 10. The result of vertical parking experiment. (a) $x-y$ path, (b) $x$ coordinate, (c) $y$ coordinate, (d) orientation angle $\Theta$, (e) driving velocity $u$, (f) driving input: $u * \cos \Theta$.

errors are adjusted to be very small by the feedback of system; that is, the designed system is stable and converges quickly. Combined with Fig. 9 (d), noting that the final parking errors are about $(x, y, \theta)=\left(180 \mathrm{~mm}, 50 \mathrm{~mm}, 1.7^{\circ}\right)$. Considering that Zhihong unmanned vehicle is equipped with a collisionavoidance system, Figs. 9 (a), (b), (c) and (d) show that there are no changing track. Therefore, the vehicle does not touch the line in the whole process. Fig. 9 (e) shows that the speed is basically constant and low, which is about $0.47 \mathrm{~m} / \mathrm{s}$. That is because of the experimental environment and safety factors. During the whole experiment, the driving input is free, and naturally, other driving input different from those in Fig. 9 (f) can also be accepted.

For vertical parking, according to the IV-B formulas, the auxiliary destinations and the final destination are calculated and marked in Fig. 10. Fig. 10 (a) shows that the curve has a certain distance from point $\mathrm{B}$, there is a slight jitter at point $\mathrm{C}$ in Figs. 10 (b), (c), (d) and (e), but the final parking result is ideal. Therefore, the designed system is stable enough and the convergence speed is ideal. Similarly, the driving input in Fig. 10 (f) is free, and the whole process is safe and collisionfree, the parking speed is not high, which is about $0.63 \mathrm{~m} / \mathrm{s}$. Noting that the final parking errors are about $(x, y, \theta)=(77$ $\mathrm{mm}, 770 \mathrm{~mm}, 2.4^{\circ}$ ), parking time is $22 \mathrm{~s}$. Compared with the garage's size, it is obvious that the vehicle can stop at the desired position accurately.
Fig. 11. The result of $45^{\circ}$ oblique parking experiment. (a) $x-y$ path, (b) $x$ coordinate, (c) $y$ coordinate, (d) orientation angle $\Theta$, (e) driving velocity $u$, (f) driving input: $u * \cos \Theta$.

For $45^{\circ}$ oblique parking, the result is shown in Fig. 11 . The theoretical path in Fig. 5 shows that the A-B-C process is difficult because the distance is short and there are many operation changes, especially the A-B process. Figs. 11 (a), (b), (c) and (d) show that the parking process has high accuracy, which further proves the designed system is efficient. Fig. 11 (e) shows that the overall average speed is about 0.48 $\mathrm{m} / \mathrm{s}$. The driving input in Fig. 10 (f) is also free. Noting that the final parking errors are about $(x, y, \theta)=(43 \mathrm{~mm}, 160$ $\mathrm{mm}, 1.3^{\circ}$ ), parking time is $25 \mathrm{~s}$. So that the control strategies and parking path are reasonable and efficient. Compared with theoretical experiments, real experiments have more reliable conclusions.

\section{Conclusion And Future Work}

This paper has proposed a simple feedback control method to guarantee the global exponential convergence rate in fully automatic parking. The continuous and piecewise smooth control inputs guarantee the reliability in real road experiment. The essential convergence of the switching algorithm depends on the horizontal velocity of vehicle $y_{0}$. The switching control algorithm and backstepping theory have been applied to deal with the stability constraints. The convergence rate problem has been solved successfully. However, some issues, such as systems with delay problems and constrained path planning [23-24], need to be researched in the future. 


\section{REFERENCES}

[1] Z. Li, B. Huang, A. Ajoudani, C. Yang, C.Y. Su, A. Bicchi, "Asymmetric bimanual control of dual-arm exoskeletons for human-cooperative manipulations," IEEE Transactions on Robotics, vol. 34, no. 1, pp. 264-271, 2017.

[2] K. Chend, Y. Zhang, and H. Chen, "Planning and control for a fully automatic parallel parking assist system in narrow parking spaces," in Proc. IEEE Int. Symp. Intell. Veh., Gold Coast, Australia, Jun. 2013, pp. 1440-1445.

[3] R. Chai, A. Tsourdos, A. Savvaris, "Two-stage trajectory optimization for autonomous ground vehicles parking maneuver," IEEE Trans. Ind. Inform., vol. 15, no. 7, pp. 3899-3909, July 2019.

[4] S. Lee, M. Kim, Y. Youm, and W. Chung, "Control of a car-like mobile robot for parking problem," in Proc. IEEE Int. Conf. Robot. Autom., Detroit, Michigan, USA, May. 1999, pp. 1-6.

[5] I. E. Paromtchik, P. Garnier, and C. Laugier, "Autonomous maneuvers of a nonholonomic vehcile," in Proc. Int. Symp. Exp. Robot., Barcelona, Spain, Jun. 1997, pp. 277-288.

[6] X. Du, and K. K. Tan, "Autonomous reverse parking system based on robust path generation and improved sliding mode control," IEEE Trans. Intell. Transp. Syst., vol. 19, no. 5, pp. 1629-1639, May. 2018.

[7] H. Gao, and D. Li, "Object classification using CNN-based fusion of vision and LIDAR in autonomous vehicle environment," IEEE Trans. Ind. Inform., vol. 14, no. 9, pp. 4224-4231, Sept. 2018.

[8] Z. Li, B. Huang, Z. Ye, M. Deng, C. Yang, "Physical human" Crobot interaction of a robotic exoskeleton by admittance control," in IEEE Transactions on Industrial Electronics, vol. 65, no. 12, pp. 9614-9624, 2018.

[9] W. He, T. Wang, and X. He, "Dynamical modeling and boundary vibration control of a rigid-flexible wing system," IEEE/ASME Transactions on Mechatronics DOI: 10.1109/TMECH.2020.2987963, Apr. 2020.

[10] M. B. Oetiker, G. P. Baker, and L. Guzzella, "A navigation-field-based semi-autonomous nonholonomic vehicle-parking assistant," IEEE Trans. Veh. Technol., vol. 58, no. 3, pp. 1106-1118, Mar. 2009.

[11] Y. Liu, W. Su, Z. Li, G. Shi, X. Chu, Y. Kang, and W. Shang, "Motor imagery based teleoperation of a dual-arm robot performing manipulation tasks," in IEEE Transactions on Cognitive and Developmental Systems, vol. 11, no. 3, pp. 414-424, 2019.

[12] Z. Li, Y. Yuan, L. Luo, et al., "Hybrid brain/muscle signals powered wearable walking exoskeleton enhancing motor ability in climbing stairs activity, "IEEE Transactions on Medical Robotics and Bionics, vol. 1, pp. 218-227, 2019.

[13] S. Zhang, Y. Dong, and Y. Ouyang, "Adaptive neural control for robotic manipulators with output constraints and uncertainties," IEEE Transactions on Neural Networks and Learning Systems, vol. 29, no. 11, pp. 5554-5564, Nov. 2018.

[14] B. Mueller, J. Deutscher, and S. Grodde, "Continuous curvature trajectory design and feedforward control for parking a car," IEEE Trans. Control Syst. Technol., vol. 15, no. 3, pp. 541-553, May 2007.

[15] D. $\mathrm{Li}$, and $\mathrm{H}$. Gao, "A hardware platform framework for an intelligent vehicle based on a driving brain," Eng., vol. 4, no. 2018, pp. 464-470, 2018.

[16] J. Fu, F. Tian, T. Chai, Y. Jing, Z. Li and C.Y. Su "Motion tracking control design for a class of nonholonomic mobile robot systems," IEEE Trans. Syst. Man. Cybern., vol. 50, no. 6, pp. 2150-2156, 2020.

[17] X. Wu, Z. Li, Z. Kan, and H. Gao, "Reference trajectory reshaping optimization and control of robotic exoskeletons for human-robot co-Manipulation," IEEE Trans. Cybern., DOI: 10.1109/TCYB.2019.2933019.

[18] H. Xiao, Z. Li, and C. L. P Chen, "Formation control of leader follower mobile robots systems using model predictive control based on neuraldynamic optimization," IEEE Trans. Ind. Electron., vol. 63, no. 9, pp. $5752-5762,2016$

[19] J. P. Laumond, P. E. Jacobs, M. Taix, and R. M. Murray, "A motion planner for nonholonomic mobile robots, "IEEE Trans. Robot Autom. vol. 10, no. 5, pp. 577-593, Oct. 1994.

[20] W. He, C. Xue, and Xinbo Yu, "Admittance-based controller design for physical human-robot interaction in the constrained task space," IEEE Transactions on Automation Science and Engineering, DOI: 10.1109/TASE.2020.2983225, Apr. 2020.

[21] Y. K. Lo, A. B. Rad, C. W. Wong, and M. L. Ho, "Automatic parallel parking," in Proc. IEEE Conf. Intell. Transp. Syst., Shanghai, China, Oct. 2003, pp. 1190-1193, Oct. 2003.

[22] Z. Li, J Li, S. Zhao, Y. Yuan, Y. Kang, C. L. P. Chen, "Adaptive neural control of a kinematically redundant exoskeleton robot using brain-machine interfaces," IEEE Transactions on Neural Networks and Learning Systems, vol. 30, pp. 3558 - 3571, 2019.

[23] H. Su, Y. Hu, H. Karimi, A. Knoll, G. Ferrigno, E. De Momi, "Improved recurrent neural network-based manipulator control with remote center of motion constraints: Experimental results," Neural Networks, vol. 131, pp. $291-299,2020$.

[24] H. Su, W. Qi, Y. Hu, H. Karimi, G. Ferrigno, E. De Momi, "An Incremental Learning Framework for Human-like Redundancy Optimization of Anthropomorphic Manipulators," IEEE Transactions on Industrial Informatics, 2020.

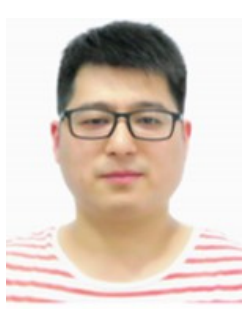

Hongbo Gao received the Ph.D. degree from Beihang University, Beijing, China, in 2016. He is currently an associate professor with the Department of Automation, School of Information Science and Technology, University of Science and Technology of China, Hefei, China. He has published over 40 journal papers, and he is the co-holder of 10 patent applications. His current research interests include unmanned system and robotics, machine learning, decision support system, intelligent driving.

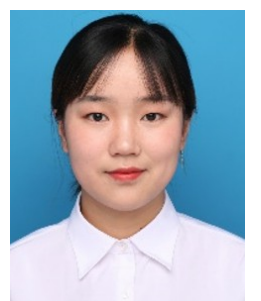

Juping Zhu received the B.S. degree in mathematics from Anhui University, Anhui, China, in 2020. She is currently pursuing the M.S. degree in control engineering with the University of Science and Technology of China, Anhui Province, China. Her current research interests include unmanned system platform and robotics, machine learning, decision support system, intelligent driving.

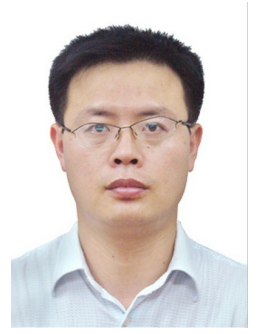

Xinde Li (M'09-SM'16) received the Ph.D. degree in control theory and control engineering from the Department of Control Science and Engineering, Huazhong University of Science and Technology, Wuhan, China, in 2007. After receiving the Ph.D. degree, he joined the School of Automation, Southeast University, Nanjing, China, where he is currently a Professor. His research interests include information fusion, object recognition, computer vision, intelligent robots, and human robot interaction.

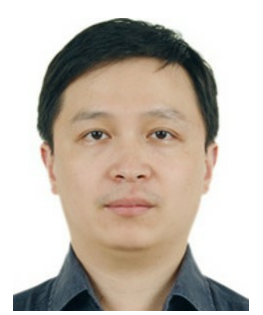

Yu Kang (M'09-SM'14) received the Dr.Eng. degree in control theory and control engineering from the University of Science and Technology of China, Hefei, China, in 2005. He is currently a Professor with the Department of Automation, University of Science and Technology of China, Anhui Province, China. He is the author or coauthor of over 60 journal papers, and he is the co-holder of 20 patent applications. His current research interests include artificial intelligence, adaptive/robust control, and variable structure control.

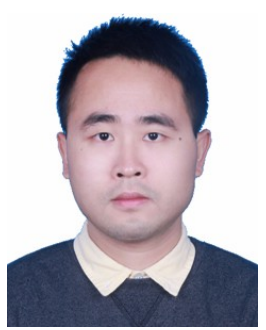

Jiehao Li received the M.Sc. degree of Control Engineering in South China University of Technology, Guangzhou, China, in 2017. He is currently pursuing a Ph.D. degree in Beijing Institute of Technology, China, and as a visiting Research Fellow, from 20192020, in Politecnico di Milano, Milano, Italy. His interests mainly include robot motion control, autonomous tracking control, model predictive control, etc.

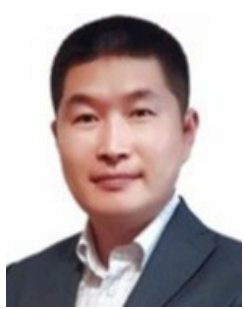

Hang Su received the Ph.D. Degree in Bioengineering from Politecnico di Milano, Milano, Italy, in 2019. He is currently working in the Department of Electronics, Information and Bioengineering (DEIB) of Politecnico Di Milano. He severs as the Associate Editor for ICRA, IROS, ICARM, ROMAN, etc. His current research interests include control and instrumentation in medical robotics, human-robot interaction, surgical robotics, deep learning, bilateral teleoperation. 